\title{
Acknowledgments
}

The production of the lantern and its standardisation within such close limits has been made possible only by the co-operation of many helpers including commercial firms. Messrs. Kelvin, Bottomley \& Baird, Ltd., have naturally taken the greatest burden, but sincere thanks are also due to Messrs. Chance Bros. \& Co., Ltd., and to Messrs. Edison Swan Electric Co., Ltd. I am indebted to Dr. W. D. Wright for much assistance with colorimetric measurements and preliminary tests, and also to Messrs. J. Guild and R. Donaldson of the Optics division of the National Physical Laboratory for their careful work in the present connection and many suggestions. My thanks are also due to Sir John Parsons, F.R.S., for the consistent encouragement which he has given, as Chairman of the Medical Research Council's Committee on Vision, to the effort to produce a satisfactory instrument.

\section{VITAL STAINING OF THE RETINA: Preliminary Clinical Note}

BY

\section{ARNOLD SORSBY}

LONDON

THE use of colouration in the study of tissues goes back to at least the sixteenth century but it is owing to the development of synthetic dyes in the second half of the last century and largely to the work of Ehrlich that vital staining has become a significant procedure. It has found applications in the study of the metabolic behaviour of tissues in tracing paths of transmission of fluids and sites of deposit of cells, and it is mainly by this approach that the conception of the reticulo-endothelial system has been elaborated by Goldmann, Kiyono and Aschoff. One obvious application, the intra ritam staining of cells for histological purposes has on the whole proved disappointing, partly because of the difficulty in fixing sections without washing the dye out. Negative results in attempts at staining the central nervous system led Goldmann to postulate the existence of a blood-brain barrier preventing dyes from reaching nervous tissue.

The conception of the blood-brain barrier gives a theoretical basis for the clinical experience that toxins circulating in the blood stream do not kill by a paralytic action on vital centres, and that toxic substances injected intrathecally are less well tolerated than when injected intravenously. The existence of a blood-brain barrier also explains the failure of therapeutic agents, such as the arsphenamides, to influence syphilitic lesions in the central 
nervous system. Were this barrier one operating as between the blood and cerebro-spinal fluid-as was suggested by the earlier work of Goldmann who found the dye he used (trypan blue) mobilised in the choroidal plexus-no great therapeutic problem would be involved, for medications could be injected intrathecally. 'That the barrier is much more complex is shown by the fact that dyes that penetrate the cerebro-spinal fluid do not necessarily stain the brain (Goldmann ${ }^{1}$ and others) and that staining of the brain can be achieved by selected dyes which do not pass into the cerebro-spinal fluid (Friedemann and Elkeles, ${ }^{2} 1931$ and 1932).

The recent recognition that central nervous tissue can be stained implies that the barrier is not absolute. Actually staining has been obtained only with basic dyes and these are all highly toxic, and it is held by some authorities ( $\mathrm{Spatz}^{3}$ and others) that such staining is supravital, occurring only in the dying animal.

These theoretical considerations have a bearing on the problem of making the normally translucent retina visible in life by vital staining. Obviously the dyes used by Goldmann and his school - the acid dyes of which trypan blue is the outstanding exampleare not applicable as they do not stain the central nervous system. Experimental work on vital staining of ocular tissues, noted in an earlier publication, ${ }^{6}$ bore out the impracticability of these dyes for staining the fundus. The possibility of using basic dyes could only be considered if it could be shown that the staining obtained with such dyes is not a supravital phenomenon, and that basic dyes of a non-toxic and locally non-irritating character are available.

1. Experimental observations.-A large number of basic dyes, such as gentian violet and other blue and green dyes, were tried experimentally in rabbits. Such staining as was obtained in the fundus was so transitory as to be doubtful, and invariably with doses giving any suggestive staining the animal collapsed. Rather similar findings had been obtained by Kikai. ${ }^{4}$ Staining of the brain as determined post-mortem was not always particularly deep, and in the course of this work the failure of the non-toxic acid dyes to stain the brain and retina was confirmed.

To overcome the inherent toxicity of the basic dyes a study was made of a number of basic dyes containing sulphonic acid radicles, as sulphonation is one of the ways in which the toxicity of substances is reduced by the body. The addition of a sulphonate radicle renders a basic dye chemically an amphoteric one -and the question remained whether this detoxicating chemical change would interfere with the properties of basic dyes to stain nervous tissues. Rhodamin dyes were also studied, as they promised to be less toxic than the pure basic dyes. As in the case with the basic dyes it was found that not all sulphonated 
dyes were equally effective as staining agents, and the toxicity of the different dyes varied largely. The first satisfactory results were obtained by Light Green S.F., a sulphonated triphenylmethane dye and Fast Acid Violet, a rhodamine dye. They were not highly toxic and gave satisfactory staining of the brain in the intact animal.

The availability of non-toxic dyes that stain the nervous tissue did not, however, solve the whole problem. With these dyes whilst effective staining of the brain could be obtained in the intact animal, staining of the fundus was, as with the pure basic dyes, of the most transient character. The retinal vessels could be seen carrying a coloured blood-stream for about two minutes, but the colouration of the fundus amounted to little more than a I ransient flushing. As the observations of Fischer ${ }^{5}$ and of Kikai that basic dyes are reduced to the leuco-base in the eye had been confirmed, the question arose whether the damaged retina would remain stained after injection. With this object experimental degeneration of the retina was induced by septojod and thermocautery according to the procedures described elsewhere. ${ }^{6}$ It was found that under such conditions an intense staining of the fundus persisting for several hours was obtained, subsequent to the transient flushing seen in the normal animal and also observed in these specially prepared animals. Rabbits, both normal and with damaged retinae-the latter being stained-were demonstrated at the January, 1937, meeting of the Section of Ophthalmology of the Royal Society of Medicine.

In subsequent work experimental degeneration of the retina was obtained by the intravenous injection of 5 c.c. of 2 per cent. sodium iodate, and the dyes used were Kiton Fast Green V and Xylene Fast Green B (tetramethyldiamino-benzhydrol-naphthalene-disulphonic acid), which proved less toxic and more satisfactory as a staining agent. The allied Wool Green S. had also been found useful.

Clinical applications.*-The clinical exploration of the possibility of vital staining of the retina had become feasible with the finding of dyes that behaved like basic dyes as far as staining of the central nervous system was concerned and were in addition non-toxic. The experimental observation that the damaged retina retained the colour suggested the possibility that the diseased retina in man might behave similarly. The dye used throughout in clinical work was Kiton Fast Green V-and after preliminary toxicity tests on monkeys the clinical dose was gradually built up. In macacus rhesus weighing 3 kilos, 15 c.c. of the 10 per cent.

\footnotetext{
* Patients with stained fundi were demonstrated at the Physiological Society in February and at the Ophthalmological Society in May, 1938.
} 


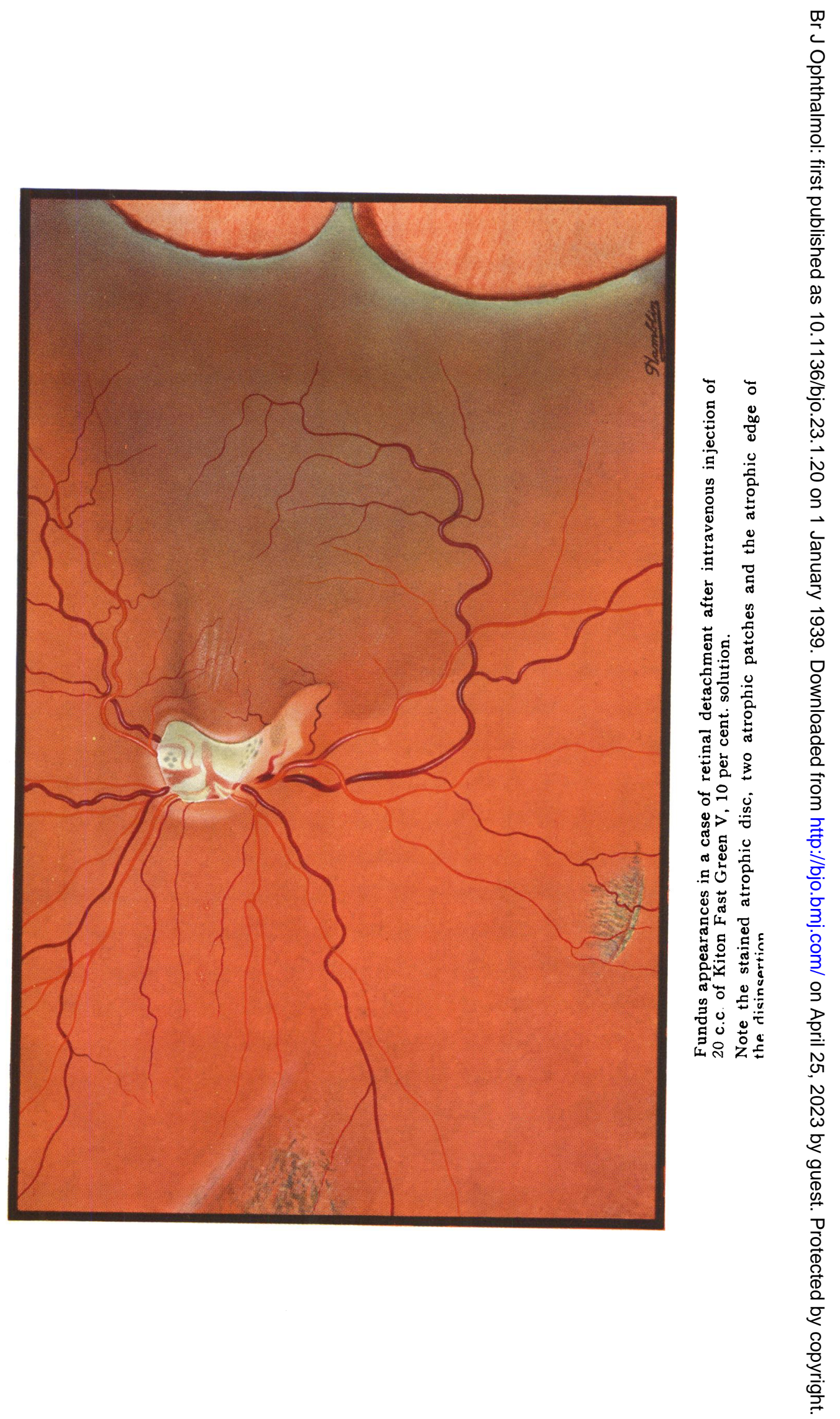


solution intravenously is non-toxic, in man an intravenous dose higher than 40 c.c. has not been used, and judging by animal experiments a higher dose is necessary to obtain the best results for staining of the retina.

At this stage it is not feasible to indicate the full clinical possibilities of vital staining of the retina. The method of use thus far evolved and a few observations are all that can be given.

(a) Method of administration.-A 10 per cent. solution of Kiton Fast Green is prepared by dissolving the dye at $60^{\circ} \mathrm{C}$., filtering twice at $35^{\circ} \mathrm{C}$., and sterilising by Tyndalisation. Twenty to thirty c.c. of this solution, warmed to body heat, are injected intravenously. After the first three to four c.c. the patient assumes a faint greenish colour, which deepens as more dye is injected. At the end of the injection the patient is deeply green, the face being greener than the rest of the body. The colour deepens during the first half hour, and begins to fade after three to four hours; generally it disappears for all practical purposes within eight to ten hours. The dye is excreted mainly through the kidneys and the urine remains coloured for two to three days.

In giving the injection attention has to be paid to several points. Owing to the dark colour of the solution special care has to be exercised in excluding air bubbles. It is also desirable to give the injection very slowly, otherwise patients are apt to vomit. Should vomiting occur the needle can be left in situ and the injection continued after a short while.

At the present, biologically standardised preparations of the dye are not yet available, so that it is necessary to test each sample of dye for toxicity.

(b) Clinical observations.-As the dye is not selective for the retina, lesions other than retinal are shown up. The blood vessels themselves do not stain, but exudates, whether choroidal or retinal, take up the dye avidly. In this, there is no advantage to be yained by using the dye, as these changes are visible ophthalmoscopically. With the doses used so far the retina in optic atrophy does not stain; in several cases all that has been observed has been a toning down of the normal red reflex. The atrophic disc itself does, however, stain green, and a suggestive finding has been obtained in one case in which vision in one eye was 6/60 and ophthalmoscopically the fundus appearances were normal; on injection, the normal eye gave no staining, the eye with defective vision gave distinctly green colouration of the disc. (In this case a dose of 40 c.c. was given.) Suggestive results have also been obtained in detached retina. In one case, the detachment, whilst not becoming green with a 30 c.c. injection, lost its normal colour, and in the subdued background a series of tears which were previously not visible, were clearly seen as 
red holes. In another case-illustrated in the colour plate-a large disinsertion which had not been observed in an exhaustive ophthalmoscopic exploration, became visible after injection of the dye (20 c.c.) the edge of the retina at the disinsertion becoming distinctly green and standing out in marked contrast to the red area uncovered by the disinsertion.

In view of the fact that normal retina decolourises all the dye that reaches it, and that colouration seems to depend upon the degree of inefficiency of the retina, it is obvious that equally deep staining is not to be expected in cases showing unequal degrees of retinal damage. Furthermore, as the dose necessary for staining experimentally damaged retina in rabbits (5-8 c.c.) suggests that higher doses than those su far employed clinically will have to be used, these preliminary clinical observations are offered as showing not the scope of vital staining of the retina, but the fact that the procedure is feasible.

My thanks are due to my collaborators in the preliminary experimental work, especially to Dr. A. Elkeles; and to the London County Council for facilities for the work. I am obliged for help in the clinical work to Dr. R. M. Stewart, Dr. E. C. Dax, Dr. H. Baker and my house surgeons at the Royal Eye Hospital, Miss E. Kennedy and Mr. J. Day. To the firms of Crookes' Laboratories, Clayton Aniline Co., Ciba, Imperial Chemical Industries, Sandoz and Etablisement Kuhlmann, I am obliged for their courtesy and helpfulness in supplying samples of dyestuffs, and to the Brit. Jl. of Ophthal. for a grant towards the expenses of this investigation.

\section{REFERENCES}

1. Goldmann, E.-Abhandl. Preuss. Akad. Wissensch., p. 1, 1913.

2. Friedemann, A. U. and Elkeles, A.-Deutsch. Med. Wochenschr., p. 194, 1931 ; Klin. Wochenschr., p. 2026, 1932.

3. Spatz, H.-Arch.f. Psychiat. und Nervenkrankh., Vol. CI, p. 267, 1934.

4. KIKAI, K.-Arch. f. Augenheilk., Vol. CIII, p. 541, 1930.

5. Fischer, F. P.-Arch. f. Augenheilk., Vols. C and CI, p. 480, p. 1929.

6. Sorsby, A., Elkeles, A., Goodhart, G. W. and Morris, I. B.-Proc. Roy. Soc. Med., Vol. XXX, p. 1271, 1937. 